\title{
Highly tunable second-harmonic generation in all-optically poled silicon nitride waveguides
}

\author{
Edgars Nitiss, ${ }^{1}$ Ozan Yakar, ${ }^{1}$ Anton Stroganov, ${ }^{2}$ and Camille-Sophie Brès ${ }^{1, *}$ \\ ${ }^{1}$ Ecole Polytechnique Fédérale de Lausanne (EPFL), Photonic Systems Laboratory (PHOSL), Lausanne CH-1015, Switzerland \\ 2 LIGENTEC SA, EPFL Innovation Park, Bâtiment L, 1024 Ecublens, Switzerland \\ ${ }^{*}$ Corresponding author: camille.bres@epfl.ch
}

Received 23 January 2020; revised 28 February 2020; accepted 28 February 2020; posted 2 March 2020 (Doc. ID 387603); published 24 March 2020

The availability of nonlinear parametric processes, such as frequency conversion in photonic integrated circuits is essential. In this contribution, we demonstrate a highly tunable second-harmonic generation in a fully complementary metal-oxide-semiconductor (CMOS)fabrication-compatible silicon nitride integrated photonic platform. We induce the second-order nonlinearity using an all-optical poling technique with the second-harmonic light generated in the fundamental mode, and a narrow quasiphase matching (QPM) spectrum by avoiding higher-order mode mixing. We are then able to broadly tune the phasematched pump wavelength over the entire $C$-band $(1540 \mathrm{~nm}$ to $1560 \mathrm{~nm}$ ) by varying the poling conditions. Fine-tuning of QPM is enabled by thermo-optic effect with the tuning slope $\Delta \lambda / \Delta T$ in our device being $113.8 \mathrm{pm} /{ }^{\circ} \mathrm{C}$. In addition, we exploit the measurable variation of the $3 \mathrm{~dB}$ QPM bandwidth to confirm how the length of the all-optically inscribed grating varies with exposure time. (c) 2020 Optical Society of America

\section{https://doi.org/10.1364/OL.387603}

Provided under the terms of the OSA Open Access Publishing Agreement

Reaching efficient wavelength conversion on an integrated photonics platform is currently an actively pursued goal in photonics. Wavelength conversion could be used in a wide variety of applications, such as frequency comb generation $[1,2]$, up-conversion detection [3], and quantum communications $[4,5]$ to name a few. The generation of new optical frequencies comes from the nonlinear optical properties of the employed material. The most frequent approach in integrated photonics for the mentioned purpose is to leverage the third-order $\left(\chi^{(3)}\right)$ nonlinearity, where new optical carriers are generated through four-wave-mixing (FWM) [6]. However, for efficient conversion through FWM high optical powers and strong confinement are necessary. Therefore, a great deal of effort is put into utilizing second-order $\left(\chi^{(2)}\right)$ nonlinearity, which is several orders of magnitude more efficient than $\chi^{(3)}$ at the same levels of optical power. $\chi^{(2)}$ also gives access to many important effects in optics, including second harmonic $(\mathrm{SH})$ generation. Unfortunately, most of the complementary metal-oxidesemiconductor (CMOS)-fabrication-compatible platforms for photonic integrated circuit development use materials that are either amorphous or centrosymmetric thus lacking secondorder nonlinearity. To enable $\chi^{(2)}$ functionality in integrated photonics several approaches have been suggested and demonstrated. One approach involves using materials that belong to the non-centrosymmetric class. Here the highest efficiency has been reached by using III-V materials such as GaAs of AlGaAs [7-9]. Unfortunately, there still is a great trade-off between high efficiency and loss in such III-V waveguides. Lithium niobate is also emerging rapidly as a material for building nonlinear integrated circuits. It has a broad transmission range and a high nonlinear coefficient [10], moreover, less than $0.2 \mathrm{~dB} / \mathrm{cm}$ loss has been demonstrated on a lithium niobate-on-insulator platform [11]. The greatest issue when using either III-V, lithium niobate, or other non-centrosymmetric material is that intermodal phase-matching has to be implemented, which for the case of $\mathrm{SH}$ generation means that the $\mathrm{SH}$ is generated in a higher-order mode while the pump is in the fundamental one [12]. This limits the conversion efficiency (CE) due to a reduced overlap integral and high loss of higher-order $\mathrm{SH}$ modes. Moreover, such an approach requires dispersion engineering in order to match the optical phases of pump and $\mathrm{SH}$ at particular wavelengths. The latter issue can be overcome by quasi-phase-matching (QPM), which is a technique for compensating the phase mismatch of pump and $\mathrm{SH}$ through creating a periodic structure in a nonlinear medium. QPM has been applied widely in waveguides possessing either $\chi^{(2)}$ or $\chi^{(3)}$ nonlinearity $[9,10,13,14]$. All aforementioned show potential for reaching high efficiencies, yet suffer from lack of flexibility and typically require complex fabrication steps involving high resolution lithography.

Considerable attention has been devoted to silicon nitride $\left(\mathrm{Si}_{3} \mathrm{~N}_{4}\right)$ which is currently one of the most mature CMOScompatible platforms for integrated photonics. $\mathrm{Si}_{3} \mathrm{~N}_{4}$ waveguides have been widely used for $\chi^{(3)}$ nonlinearity with the demonstration of optical frequency comb generation [1,2,15], ultra-low loss resonators [16-18], entangled photon-pair generation [19], as well as low pulse energy supercontinuum generation in the near [20] and mid-infrared [21]. Recently we showed that efficient QPM and SH generation can be achieved in $\mathrm{Si}_{3} \mathrm{~N}_{4}$ waveguides by using all-optical poling [22]. During all-optical poling an effective $\chi^{(2)}$ builds up spontaneously in the presence of a high intensity coherent pump and weak $\mathrm{SH}$ 
due to the coherent photogalvanic effect $[23,24]$. Additional recent studies of all-optical poling of $\mathrm{Si}_{3} \mathrm{~N}_{4}$ waveguides have confirmed the presence of an effective $\chi^{(2)}$ grating and given insight into its formation process [25-28]. The key strength of the used approach is that it allows automatic QPM of fundamental modes at pump and $\mathrm{SH}$ without the need to engineer the dispersion of the waveguide. Despite the latter, the parameters of the employed waveguide can have significant influence on QPM properties. We have reported indications of energy transfer to SH modes other than the fundamental one. This is suggested to be a result of mode distortions or mode mixing experienced in the waveguide's bends leading to a broadening of the expected QPM spectral response. Mode mixing can be avoided by reducing the mode overlap between the fundamental mode of the $\mathrm{FH}$ with higher-order modes at $\mathrm{SH}$ in the waveguide bends [27]. Importantly, the all-optical poling introduces second-order nonlinearity in the waveguide without any obvious change of other properties such as propagation loss. In this contribution we demonstrate the flexible broadband spectral tuning capabilities of QPM in all-optically poled $\mathrm{Si}_{3} \mathrm{~N}_{4}$ waveguides as well as highlight the importance of thermal stability of the chip during the all-optical poling process.

The $\mathrm{Si}_{3} \mathrm{~N}_{4}$ waveguide used in this study has dimensions of $0.57 \mu \mathrm{m} \times 0.81 \mu \mathrm{m}$ and was fabricated by LIGENTEC using its AN800 technology platform [29]. The $81 \mathrm{~mm}$ long waveguide is folded in nine meanders on a $10 \mathrm{~mm} \times 5 \mathrm{~mm}$ chip by using curved sections with a radius of $75 \mu \mathrm{m}$. The waveguide dimensions were chosen such that $\mathrm{SH}$ is generated in the fundamental mode, and QPM broadening due to mode mixing would not be present [27]. The waveguide was poled using an optical setup described in Ref. [22]. Here the continuous wave $(\mathrm{CW})$ light from a coherent tunable light source in the C-band is shaped by a Mach-Zehnder modulator into a 1-ns $5-\mathrm{MHz}$ pulse train and then amplified in two consecutive stages. This pulsed pump is then coupled into the waveguide using a lensed fiber. The output light of the chip is collected using a $20 \times$ microscope objective and delivered to pump and $\mathrm{SH}$ photodetectors using dichroic mirrors. The light propagation loss is measured to be $0.33 \mathrm{~dB} / \mathrm{cm}$ at $1550 \mathrm{~nm}$ and $0.73 \mathrm{~dB} / \mathrm{cm}$ at $775 \mathrm{~nm}$. During the all-optical poling procedure, the temperature of the chip is kept constant at $T_{P}=30^{\circ} \mathrm{C}$ using a PID controller and a Peltier module. In Fig. 1 (a) the growth of type-0 SH CE versus time is shown when a high intensity TE polarized coherent pump at $1550 \mathrm{~nm}$ with maximum peak-power of $29.4 \mathrm{~W}$ is coupled into the waveguide. As evident, the $\mathrm{SH}$ intensity grows and saturates at $0.5 \% / \mathrm{W}$ in less than $10 \mathrm{~min}$. The efficiency could be further increased by raising the coupled pump power during poling [27]. We interrupt the all-optical poling process at times indicated by red circles in Fig. 1(a) to study the properties of the QPM. At each interruption instant, the $\mathrm{SH}$ spectral response induced by the low $\mathrm{CW}$ probe was recorded around the pump wavelength. The CE is fit with a sinc-squared function as shown by the lines in Figs. 1(b)-1(e) according to the following equation:

$$
\mathrm{CE}=\frac{\left(\omega L_{g} \chi_{\mathrm{eff}}^{(2)}\right)^{2}}{2 \varepsilon_{o} c^{3} n_{\omega}^{2} n_{2 \omega} S}\left(\frac{\sin \left(\Delta \beta L_{g} / 2\right)}{\Delta \beta L_{g} / 2}\right)^{2},
$$

where $\omega$ is the pump frequency, $L_{g}$ is the grating length, $\chi_{\text {eff }}^{(2)}$ is the effective nonlinearity, $n_{\omega}$ and $n_{2 \omega}$ are the refractive indexes
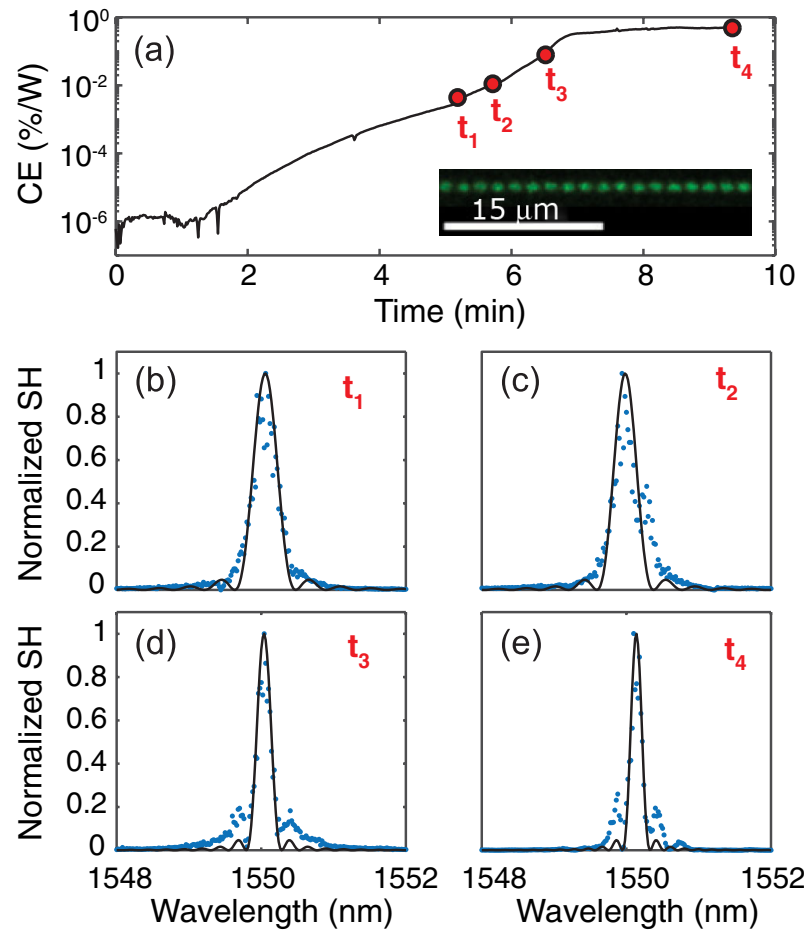

Fig. 1. (a) Growth of CE during all-optical poling as a function of time. The red marks indicate instants $\left(t_{1}, t_{2}, t_{3}\right.$, and $\left.t_{4}\right)$ at which the poling is interrupted for CE spectra measurements. Inset: TPM image of the grating at time $t_{4}$. (b)-(e) Measured and fitted CE spectra (dots) at interrupt instants $t_{1}, t_{2}, t_{3}$, and $t_{4}$, respectively.

Table 1. Summary of Parameters Extracted from CE Spectra Measurement and Fit

\begin{tabular}{lcccc}
\hline & $\boldsymbol{t}_{\mathbf{1}}$ & $\boldsymbol{t}_{\mathbf{2}}$ & $\boldsymbol{t}_{\mathbf{3}}$ & $\boldsymbol{t}_{\boldsymbol{4}}$ \\
\hline$L_{g}(\mathrm{~mm})$ & 20.3 & 21.8 & 34.8 & 40.8 \\
$B W_{3 \mathrm{~dB}}(\mathrm{~nm})$ & 0.38 & 0.35 & 0.22 & 0.17 \\
$\Lambda(\mu \mathrm{m})$ & 3.24 & 3.24 & 3.24 & 3.24 \\
$\operatorname{Max} \mathrm{CE}(\% / \mathrm{W})$ & 0.005 & 0.01 & 0.08 & 0.49 \\
$\chi_{\text {eff }}^{(2)}(\mathrm{pm} / \mathrm{V})$ & 0.011 & 0.015 & 0.024 & 0.058 \\
\hline
\end{tabular}

at pump and $\mathrm{SH}$ wavelengths, respectively. $S$ is the effective area, and $\Delta \beta$ is the propagation constant mismatch [30]. The $\Delta \beta$ after all-optical poling of the waveguide can be written as

$$
\Delta \beta=\beta_{2 \omega}-2 \beta_{\omega}-\frac{2 \pi}{\Lambda},
$$

where $\Lambda$ is the grating period, and $\beta_{2 \omega}$ and $\beta_{\omega}$ are the propagation constants at the $\mathrm{SH}$ and fundamental harmonic, respectively [31]. The fits of CE spectral responses allow for the extraction of $\Lambda, L_{g}$, as well as $\chi_{\mathrm{eff}}^{(2)}$. The obtained data is summarized in Table 1.

As evident from Figs. 1(b)-1(e), the bandwidth of the $\mathrm{SH}$ spectrum $\left(\mathrm{BW}_{3 \mathrm{~dB}}\right)$ becomes narrower with poling time. This narrowing of the bandwidth is due to an increase of the grating length as the waveguide poling efficiency increases [27]. At time $t_{4}$ we performed two-photon microscopy (TPM) imaging of the $\chi^{(2)}$ grating [see inset Fig. 1(a)]. The retrieved $\chi^{(2)}$ grating period from TPM measurements is estimated to 

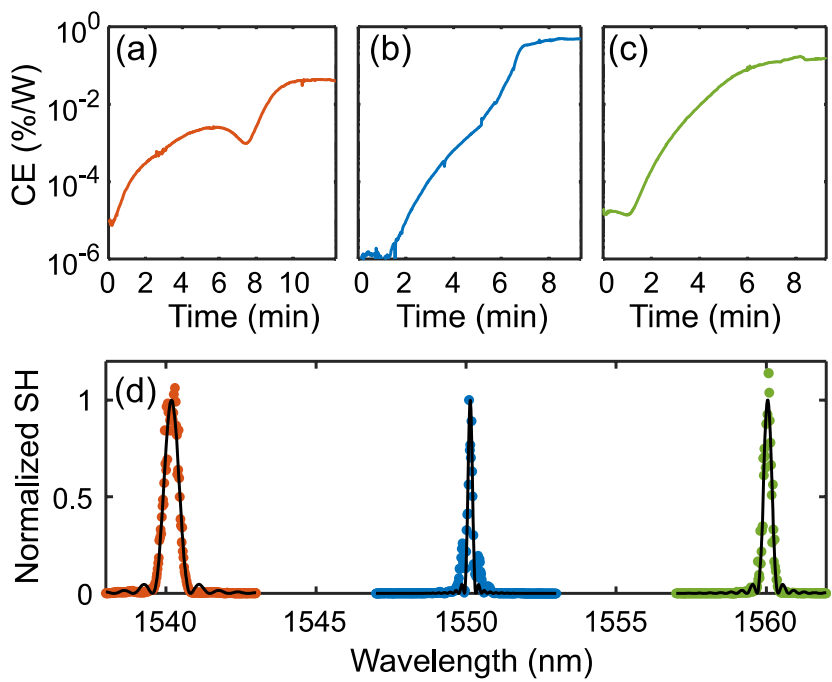

Fig. 2. (a) -(c) CE as a function of time for a pump wavelength of $1540 \mathrm{~nm}, 1550 \mathrm{~nm}$, and $1560 \mathrm{~nm}$, respectively. The used pump peak-power during all-optical poling was $26.2 \mathrm{~W}, 29.4 \mathrm{~W}$, and $28.1 \mathrm{~W}$, respectively. (d) Corresponding normalized CE spectra measured after each poling. Dots are the experimental data, lines are sinc-squared function fits.

be $3.34 \pm 0.10 \mu \mathrm{m}$, in very good agreement with the values obtained from the fitting of the CE spectra (see Table 1).

The broadband tuning of QPM is enabled by poling the waveguide at different wavelengths, as the $\Lambda$ will automatically adjust due to the change in propagation constants. After poling the waveguide using a $1550 \mathrm{~nm}$ pump and measuring the CE spectrum, we repeat the all-optical poling process in the same waveguide using a $1540 \mathrm{~nm}$ and a $1560 \mathrm{~nm}$ pump with peak powers of $26.2 \mathrm{~W}$ and $28.1 \mathrm{~W}$, respectively. The recorded CE in $\% / \mathrm{W}$ at each poling wavelength is plotted in Fig. 2(a). As evident, $\mathrm{SH}$ saturation in each case is reached in a time span of less than $10 \mathrm{~min}$. During each consecutive all-optical poling at new wavelength the previously inscribed grating is erased, and no response at the previous poling wavelength is measurable.

From Figs. 2(a)-2(c), it is evident that even small differences in used poling pump power has significant influence on the saturation CE. It is once again confirmed that the maximal reachable efficiency grows exponentially with the increase in used pump peak power [27]. The maximum CE is therefore lowest $(0.0023 \% / \mathrm{W})$ for a $1540 \mathrm{~nm}$ poling. We also obtain $0.16 \% / \mathrm{W}$ at $1560 \mathrm{~nm}$ and $0.49 \% / \mathrm{W}$ at $1550 \mathrm{~nm}$. In Fig. 2(d) we plot the $\mathrm{SH}$ spectral responses after each poling step. Clearly, QPM inside the waveguide adjusts to match to the pump used during poling, and we did not measure any signature of the previously inscribed grating. In addition to the variation in maximum CE, we can also measure a change in the $3 \mathrm{~dB}$ bandwidth which follows the same principle observed in Fig. 1.

Broad tuning of the QPM conditions can therefore be obtained by re-poling of the waveguide. We show here coverage of the entire C-band, limited only by the bandwidth of the amplifier. We expect that a much broader tuning range can be reached. For example, successful poling of $\mathrm{SiN}$ waveguides has also been demonstrated using $1064 \mathrm{~nm}$ light source [25]. In addition, we can finely tune the QPM after poling by leveraging the thermo-optic effect. As a demonstration, we first poled the waveguide at $30^{\circ} \mathrm{C}$ using a $1560 \mathrm{~nm}$ pump wavelength. We then
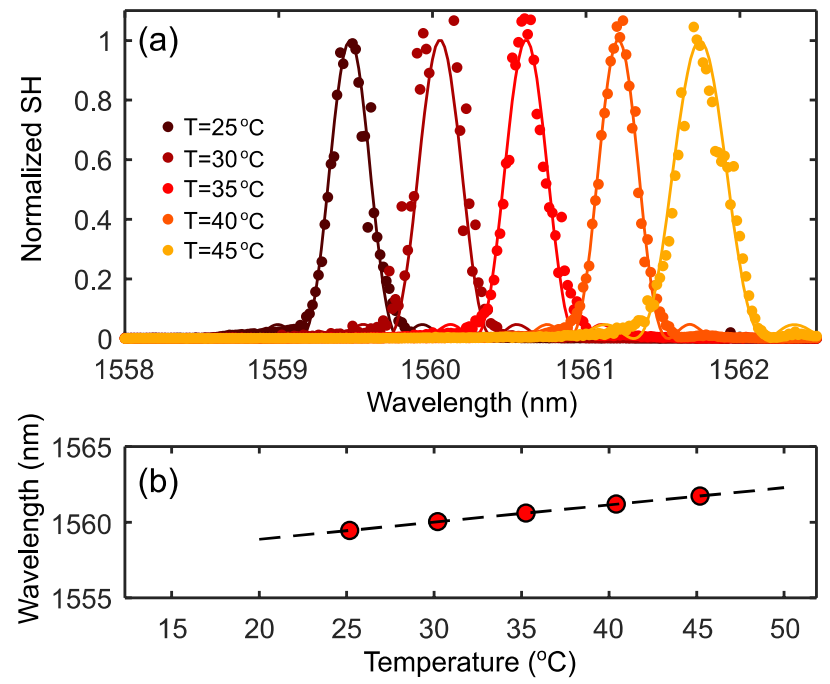

Fig. 3. (a) Normalized CE spectra for five different temperatures from $25^{\circ} \mathrm{C}$ to $45^{\circ} \mathrm{C}$. The waveguide was initially poled at $1560 \mathrm{~nm}$ and $30^{\circ} \mathrm{C}$. Dots are the experimental data, lines are sinc-squared function fits. (b) Extracted wavelength versus temperature dependence. The dashed line is a linear fit.

perform probing of the QPM spectrum with a CW wave for different temperature settings. In Fig. 3(a), the normalized $\mathrm{SH}$ spectra for five temperatures, from $25^{\circ} \mathrm{C}$ to $45^{\circ} \mathrm{C}$, are plotted. Note that we performed the five measurements in random order. Also, while the normalized spectra are shown here, we did not measure any significant variations in the actual maximum $\mathrm{CE}$ value. From sinc-squared fits of the experimental data, we extract the wavelength at which the maximal CE takes place. The measured phase-matched pump wavelength as a function of temperature is plotted in Fig. 3(b). The tuning slope $\Delta \lambda / \Delta T$ from the linear fit is calculated to be $113.8 \mathrm{pm} /{ }^{\circ} \mathrm{C}$.

The thermal tuning is enabled by the thermo-optic effect in the waveguide's cladding and core. Given the phase matching condition in Eq. (2), and assuming small variations in the refractive index difference as the temperature is changed by $\Delta T$, the change in maximal CE wavelength $\Delta \lambda$ with respect to the initial pump wavelength $\lambda_{P}$ can be calculated using the following equation:

$$
\Delta \lambda=\frac{\lambda_{P}}{\Delta n_{\mathrm{eff}}} \frac{\partial\left(\Delta n_{\mathrm{eff}}\right)}{\partial T} \Delta T
$$

where $\Delta n_{\text {eff }}$ is the absolute difference between the effective refractive indexes at $\mathrm{FH}$ and $\mathrm{SH}$ wavelengths. In a waveguide with cross-section $0.57 \mu \mathrm{m} \times 0.81 \mu \mathrm{m}$ initially poled using a pump wavelength of $1560 \mathrm{~nm}$, the $\Delta n_{\text {eff }}$ is calculated to be 0.2594 . Based on Eq. (3) and the experimentally measured slope, the $\partial\left(\Delta n_{\text {eff }}\right) / \partial T$ is estimated at $1.89 \cdot 10^{-5} 1 / \mathrm{K}$. The thermal tuning slope will vary with the dimensions of the alloptically poled waveguide. The size of the waveguide determines the $\partial\left(\Delta n_{\text {eff }}\right) / \partial T$ as well as $\Delta n_{\text {eff. }}$. For waveguides with a larger cross-section, the $\Delta n_{\text {eff }}$ will be smaller, while the $\partial\left(\Delta n_{\text {eff }}\right) / \partial T$ will become increasingly determined by the thermo-optic properties of the core. It is also important to note that increasing the operating temperature of the waveguide will reduce the lifetime of the inscribed grating [27]. In addition, we observed that a change in coupling conditions of $1 \mathrm{~dB}$ (due to in-coupling 
fluctuations) can significantly influence the measured CE as well as the poling curve. We believe this is due to temperature variations despite having temperature stabilization. Indeed for these measurements, and given the $\sim 0.3 \mathrm{~nm}$ bandwidth of the QPM spectrum, only $1 \operatorname{deg} \mathrm{C}^{\circ}$ variation in temperature causes a $3 \mathrm{~dB}$ reduction in the $\mathrm{SH}$ response. The thermal stabilization of the sample holder might be too slow under such small temperature changes, which can explain oscillations in CE sometimes observed during poling [see Fig. 3(a)]. Thermal stability of the chip during the all-optical poling process is therefore of critical importance to avoid drifts and overall decrease in efficiency.

To conclude, we show a nanophotonic all-optically poled $\mathrm{Si}_{3} \mathrm{~N}_{4}$ waveguide with induced second-order nonlinearity for tunable and efficient $\mathrm{SH}$ generation. We measured a $\mathrm{CE}$ of $0.49 \% / \mathrm{W}$ in a waveguide allowing for narrow sinc-shaped QPM spectra. The used approach is simple to implement and does not require traditionally applied complex nanostructuring of waveguides for obtaining QPM at the necessary wavelength. Owing to the high quality of QPM, we observed the evolution of the $\mathrm{CE}$ spectrum during poling, which narrows and increases in efficiency, a clear confirmation that the grating grows in length and strength during the poling process. Broadband tuning of QPM is enabled by changing the pump wavelength used during poling, owing to the highly flexible and reconfigurable nature of the underlying physical principle. We show a broad tunability over the entire C-band, only limited by the bandwidth of the EDFA. Over this entire tuning range, the CE spectrum maintains its sinc-shape. Fine tuning of QPM after poling is enabled by thermo-optic effects. The tuning slope $\Delta \lambda / \Delta T$ in our device is measured to be $113.8 \mathrm{pm} /{ }^{\circ} \mathrm{C}$ which is of the same order of magnitude as achieved in lithium niobate [12,31].

Funding. European Research Council (PISSARRO ERC-2017-CoG 771647).

Disclosures. The authors declare no conflicts of interest.

\section{REFERENCES}

1. T. J. Kippenberg, R. Holzwarth, and S. A. Diddams, Science 332, 555 (2011).

2. A. L. Gaeta, M. Lipson, and T. J. Kippenberg, Nat. Photonics 13, 158 (2019).

3. L. Lehmann, L. Grossard, L. Delage, F. Reynaud, M. Chauvet, and F. Bassignot, Opt. Express 27, 19233 (2019).

4. D. Grassani, S. Azzini, M. Liscidini, M. Galli, M. J. Strain, M. Sorel, J. E. Sipe, and D. Bajoni, Optica 2, 88 (2015).

5. F. Mazeas, M. Traetta, M. Bentivegna, F. Kaiser, D. Aktas, W. Zhang, C. A. Ramos, L. A. Ngah, T. Lunghi, É. Picholle, N. BelabasPlougonven, X. Le Roux, É. Cassan, D. Marris-Morini, L. Vivien, G. Sauder, L. Labonté, and S. Tanzilli, Opt. Express 24, 28731 (2016).

6. C. J. Krückel, V. Torres-Company, P. A. Andrekson, D. T. Spencer, J. F. Bauters, M. J. R. Heck, and J. E. Bowers, Opt. Lett. 40, 875 (2015).
7. L. Chang, A. Boes, P. Pintus, J. D. Peters, M. Kennedy, X.-W. Guo, N. Volet, S.-P. Yu, S. B. Papp, and J. E. Bowers, APL Photon. 4, 036103 (2019).

8. L. Chang, A. Boes, X. Guo, D. T. Spencer, M. J. Kennedy, J. D. Peters, N. Volet, J. Chiles, A. Kowligy, N. Nader, D. D. Hickstein, E. J. Stanton, S. A. Diddams, S. B. Papp, and J. E. Bowers, Laser Photon. Rev. 12, 1800149 (2018).

9. N. Morais, I. Roland, M. Ravaro, W. Hease, A. Lemaître, C. Gomez, S. Wabnitz, M. De Rosa, I. Favero, and G. Leo, Opt. Lett. 42, 4287 (2017).

10. J. E. Bowers, J. Peters, L. Wang, L. Chang, N. Volet, and Y. Li, Optica 3, 531 (2016).

11. C. Wang, M. Zhang, X. Chen, M. Bertrand, A. Shams-Ansari, S. Chandrasekhar, P. Winzer, and M. Lončar, Nature 562, 101 (2018).

12. R. Luo, Y. He, H. Liang, M. Li, and Q. Lin, Optica 5, 1006 (2018).

13. C. C. Kores, P. Mutter, H. Kianirad, C. Canalias, and F. Laurell, Opt. Express 26, 33142 (2018).

14. C. Wang, C. Langrock, A. Marandi, M. Jankowski, M. Zhang, B. Desiatov, M. M. Fejer, and M. Lončar, Optica 5, 1438 (2018).

15. H. Guo, C. Herkommer, A. Billat, D. Grassani, C. Zhang, M. H. P. Pfeiffer, W. Weng, C.-S. Brès, and T. J. Kippenberg, Nat. Photonics 12, 330 (2018).

16. Y. Xuan, Y. Liu, L. T. Varghese, A. J. Metcalf, X. Xue, P.-H. Wang, K. Han, J. A. Jaramillo-Villegas, A. Al Noman, C. Wang, S. Kim, M. Teng, Y. J. Lee, B. Niu, L. Fan, J. Wang, D. E. Leaird, A. M. Weiner, and M. Qi, Optica 3, 1171 (2016).

17. J. Liu, A. S. Raja, M. Karpov, B. Ghadiani, M. H. P. Pfeiffer, B. Du, N. J. Engelsen, H. Guo, M. Zervas, and T. J. Kippenberg, Optica 5, 1347 (2018).

18. X. Ji, F. A. S. Barbosa, S. P. Roberts, A. Dutt, J. Cardenas, Y. Okawachi, A. Bryant, A. L. Gaeta, and M. Lipson, Optica 4, 619 (2017).

19. S. Ramelow, A. Farsi, S. Clemmen, D. Orquiza, K. Luke, M. Lipson, and A. L. Gaeta, "Silicon-nitride platform for narrowband entangled photon generation," arXiv:1508.04358 (2015).

20. A. R. Johnson, A. S. Mayer, A. Klenner, K. Luke, E. S. Lamb, M. R. E. Lamont, C. Joshi, Y. Okawachi, F. W. Wise, M. Lipson, U. Keller, and A. L. Gaeta, Opt. Lett. 40, 5117 (2015).

21. D. Grassani, E. Tagkoudi, H. Guo, C. Herkommer, F. Yang, T. J. Kippenberg, and C.-S. Brès, Nat. Commun. 10, 1553 (2019).

22. A. Billat, D. Grassani, M. H. P. Pfeiffer, S. Kharitonov, T. J. Kippenberg, and C. S. Brès, Nat. Commun. 8, 1016 (2017).

23. D. Z. Anderson, V. Mizrahi, and J. E. Sipe, Opt. Lett. 16, 796 (1991).

24. E. M. Dianov and D. S. Starodubov, Quantum Electron. 25, 395 (1995).

25. M. A. G. Porcel, J. Mak, C. Taballione, V. K. Schermerhorn, J. P. Epping, P. J. M. van der Slot, and K.-J. Boller, Opt. Express 25, 33143 (2017).

26. D. D. Hickstein, D. R. Carlson, H. Mundoor, J. B. Khurgin, K. Srinivasan, D. Westly, A. Kowligy, I. I. Smalyukh, S. A. Diddams, and S. B. Papp, Nat. Photonics 13, 494 (2019).

27. E. Nitiss, T. Liu, D. Grassani, M. Pfeiffer, T. J. Kippenberg, and C.-S. Brès, ACS Photon. 7, 147 (2019).

28. D. Grassani, M. H. P. Pfeiffer, T. J. Kippenberg, and C.-S. Brès, Opt. Lett. 44, 106 (2019).

29. LIGENTEC, https://www.ligentec.com/.

30. C. Wang, C. Langrock, A. Marandi, M. Jankowski, M. Zhang, B. Desiatov, M. M. Fejer, and M. Lončar, Optica 5, 1438 (2018).

31. M. M. Fejer, G. A. Magel, D. H. Jundt, and R. L. Byer, IEEE J. Quantum Electron. 28, 2631 (1992). 